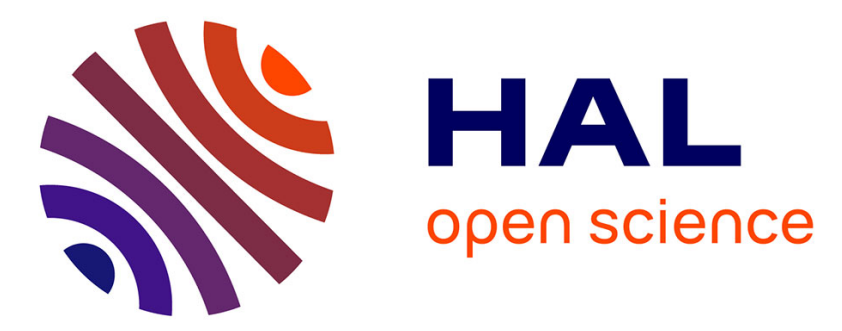

\title{
Reliability increasing through networked cascade control structure - consideration of quasi-redundant subsystems
}

Jan Galdun, Jean-Marc Thiriet, Jan Ligus, Jan Sarnovsky

\section{To cite this version:}

Jan Galdun, Jean-Marc Thiriet, Jan Ligus, Jan Sarnovsky. Reliability increasing through networked cascade control structure - consideration of quasi-redundant subsystems. IFAC WC 2008 - 17th IFAC World Congress, Jul 2008, Séoul, South Korea. pp.6839-6844. hal-00349419

\section{HAL Id: hal-00349419 https://hal.science/hal-00349419}

Submitted on 30 Dec 2008

HAL is a multi-disciplinary open access archive for the deposit and dissemination of scientific research documents, whether they are published or not. The documents may come from teaching and research institutions in France or abroad, or from public or private research centers.
L'archive ouverte pluridisciplinaire HAL, est destinée au dépôt et à la diffusion de documents scientifiques de niveau recherche, publiés ou non, émanant des établissements d'enseignement et de recherche français ou étrangers, des laboratoires publics ou privés. 


\title{
Reliability increasing through networked cascade control structure - consideration of quasi-redundant subsystems
}

\author{
Ján Galdun*,**, Jean-Marc Thiriet*, \\ Ján Liguš**, Ján Sarnovský** \\ * Laboratoire GIPSA-Lab (GIPSA-Lab UMR 5216 CNRS-INPG-UJF) \\ BP 46, F-38402 Saint Martin d'Hères Cedex \\ (e-mail:jan.galdun@tuke.sk,jean-marc.thiriet@ujf-grenoble.fr) \\ ** Department of Cybernetics and Artificial Intelligence, Technical University of Košice, \\ Letná 9, SK-04012 Košice \\ (e-mail:jan.galdun@tuke.sk,jan.ligus@tuke.sk,jan.sarnovsky@tuke.sk)
}

\begin{abstract}
The paper presents common cascade control architecture where specific kind of redundancy could be considered. There are different approaches how to increase the reliability of networked control systems. Common approach uses redundant components in control system i.e. passive or active redundancy. We deal with quasi-redundant subsystems (shared redundancy) which could be very useful when critical failure appears. This type of redundancy offers several important advantages such as minimizing the number of components as well as reliability increasing. The example of a four-rotor minihelicopter is presented where a cascade control architecture is considered. The main contribution of this paper is that it helps to determine the applications where quasi-redundant subsystems are a good solution to remain in a significant reliability level even if critical failure appears. (Copyright IFAC 2008).
\end{abstract}

Keywords: Networked control systems, Cascade control architecture, Reliability analysis, low cost, quasi (shared) redundancy.

\section{INTRODUCTION}

Different methods or approaches for control systems' reliability improvement are developed in order to be applied to specific subsystems or to deal with dependencies among subsystems. To be able to obtain relevant results of reliability evaluations for complex systems, it is necessary to describe the maximum of specific dependencies within the studied system and their influences on the system reliability.

As it was mentioned above, several different approaches exist in order to increase the systems reliability. A classical technique consists in designing a fault-tolerant control (Spooner et al. 1997) where the main aim is to propose a robust control algorithm. Guenab and others in (Guenab et al., 2006) deal with this approach and reconfiguration strategy in complex systems, too.

On the other side is the design of reliable control architectures. Probably the most used technique is to consider the redundant components which enlarge the system structure and its complexity too. Active and passive redundancy is the simplest way how to improve dependability attributes of the systems such as reliability, maintainability, availability, etc (Laprie et al., 1992). However, as it was mentioned the control structure turns to be more complex due to an increasing number of components as well as number of possible dependencies among components.
The paper introduces several networked control architectures based on cascade control structure. The cascade structure was chosen purposely due its advantages. This structure is widely used in industrial applications thanks to positive results for quality of control which are already described and generally known (Brosilow et al. 2002). On the other side it offers some possibilities of system reliability improvement. There are potentially redundant components as controllers (primary, secondary). If more than one network is implemented we could them consider as potentially redundant subsystems too. Finally if the physical system allows it, it is possible to profit from sensors. The cascade structure and other features are introduced in more details in part 3.

The paper is organised as follows. After bring closer the research background is introduced the basic structure and principle of the cascade control (further only CC). In the next part are presented networked topologies considered in CC structure as well as a 4-rotor mini-helicopter model and its networked CC structure. Using Petri nets were prepared the models of these NCCS with different topologies. These models were simulated whereby reliability of these different structures was evaluated. Finally, are proposed the simulation results and a short conclusion.

\section{RESEARCH BACKGROUND}

Control architecture design approach was taken into account by Wysocki, Debouk and Nouri (Wysocki et al., 2004). They present shared redundancy as parts of systems (subsystems) 
which could replace another subsystem in case of its failure. This feature is conditioned with the same or similar function of the subsystem. Wysocki et al. introduce the shared redundant architecture in four different examples illustrated on "X-by-Wire" systems used in automotive applications. Presented results shown advantages of this approach in control architecture design.

As it was abovementioned, Guenab (Guenab et al., 2006) and (Spooner et al. 1997) are interested in fault-tolerant approach. With regard to important advantages of shared redundancy described by Wysocki, we have decided to study this approach in more details and describe the influence of the specific feature to final reliability of the studied system.

Hence, in further text we will deal with the system where basic structure naturally offers implementation of "shared redundancy" approach. The important feature appears when a quasi-redundant component replaces the function of another one to prevent a critical failure but its reliability is decreased. Thus, the influence of dynamic reliability decreasing during the system mission to its final reliability will be shown. Thus, we will offer helpful results to show limits of the shared redundancy approach in the case where the main aim is to save the system when a critical failure occurs.

\section{REDUNDANT SUBSYSTEMS AND CASCADE CONTROL ARCHITECTURE}

\subsection{Redundant subsystems - basic types}

The primary aim of redundant components is to compensate the component failure in order to achieve its mission. There are two basic types of redundancy (Pimentel et al., 2002):

- Passive (cold),

- Active (warm/hot).

Passive redundant component starts operate at the moment when the primary component has failed. This redundancy is often conditioned by the component initialization (in the worst case by the system restart) which could not be applied in some critical control system application.

On the contrary, active redundant components are in operational mode at the same time the primary component is working. Two cases of life time of the system with active redundant components can be envisaged:

- the first case is when the primary component failed whereas the redundant component continues accomplishes its mission,

- the second case is when the redundant component failed first.

We can suppose that life mission time of the system with passive redundant components $t_{\text {plife }}$ could be longer than life time of the system with active redundancy $t_{\text {alife }}$. However, the mentioned problem with component initialization could decrease the number of application where this type of redundancy could be implemented, this depends on the PFD (Probability of Failure on Demand).

In further text we considered another type of redundant components which are not primary determined as redundant but they are able to replace some mission if it is urgently required. This type of redundancy is referred as shared redundancy (Wysocki et al., 2004).

\subsection{Active vs. shared redundancy - quasi-redundant subsystems}

In the similar sense as shared redundancy (Wysocki et al., 2004) we use term quasi-redundant components in networked cascade control structure. These parts of the system are not primary redundant. Each quasi-redundant part accomplishes its primary mission when the system is in its nominal state. However its functionality allows compensate the failure of another subsystem with similar mission.

We can see that quasi-redundant components are not primary determined as active redundant subsystem because each one has its own mission which must be accomplished. Only in case of failure this type of redundancy should be used.

In order to profit from quasi-redundant architecture the existing hardware components are used. This paper presents a simple networked cascade structure which is naturally composed of potential quasi-redundant components, for example the controllers. When a networked control structure is composed of more than one network then we could use shared redundancy approach too.

\subsection{Networked cascade control architecture}

The conventional cascade control structure is often used in industrial applications thanks to its important features which improve the quality of control. With using cascade control structure there are several constraints (Brosilow et al., 2002). The main condition requires that controlled system must contain subsystem (secondary subsystem $F_{S}(s)-$ Fig. 1) that directly affect to primary system $\mathrm{F}_{\mathrm{P}}(\mathrm{s})$.

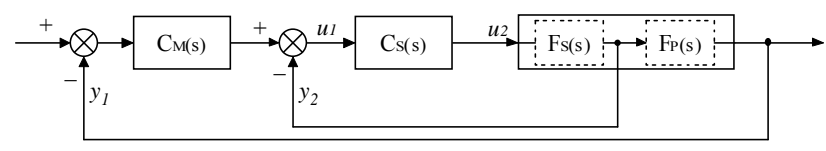

Fig. 1 Main structure of the cascade control

Usually for secondary subsystems there is a condition of faster dynamics than primary process. This condition must not be fulfilled (Brosilow et al., 2002) however, some modifications of conventional cascade structure (Fig. 2) and control laws must be provided. 


\section{CONTRIBUTION OF THE NCC STRUCTURE FOR CONTROL SYSTEMS RELIABILITY}

As it was mentioned above, using networked cascade control structure for control systems allows profit from several advantages. In this approach there are two points of view:

- Quality of control - QoC

- Reliability aspect of the networked cascade control (NCC) structure with quasi-redundant parts of the controlled system

The first aspect relates with performance parameters of the controlled system such as time of regulation, overshooting, etc. Using CC approach could improve these parameters. For example, faster disturbance elimination of the controlled system can lead to positive effect on performance parameters (Brosilow et al., 2002).

The second aspect that we would like to take profit from is the possibility which offer NCC structure in term of reliability of the system - quasi-redundant components.

\subsection{Quasi-redundant components in NCC structure}

In figure 4 is shown a networked implementation of the basic cascade structure. This control architecture is composed of two sensors, two controllers (Master, Slave) and one actuator which are connected to the network. There are two hardware components which allow implement the shared redundancy approach. The controllers follow their primary mission, stabilization and performance optimization of the controlled system. Therefore, in regard to the same hardware it allows share the computing capacity and executes different tasks. Thus, in both controllers could be implemented both control tasks - for primary and secondary subsystems.

In non-failure mode the primary task is executed in both controllers. However, in case of controller's failure (primary or secondary) non-failed controller starts execute both tasks and computes actuating value for primary as well as secondary subsystem. In this case we can suppose two scenarios.

The first one supposes that controller is able to execute all necessary algorithms within required sample periods (Fig. 2a). In this case the behavior of quasi-redundant component is identical as in case of active redundant components. Thus, in case of failure one of the components the second one takes care about its mission until its failure.

In figure $2 \mathrm{~b}$ is shown second case when time to execute both necessary tasks is grater than required sample period. Thus, controller will cause the delays which have significant influence to system stability (Galdun et al. 2007), (Ligušová et al., 2004). However, this delay is known what allows partially compensating by using several methods (Nicolescu, 1997).

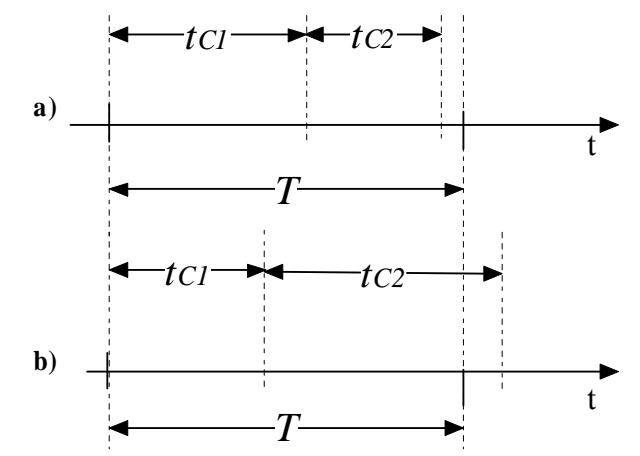

Fig. 2 Possible scenarios for quasi-redundant controllers

We can suppose that system destabilization will not occur immediately after first delay or that we are able to compensate this delay for some time interval. Thus, quasiredundant controller does not failed immediately but its reliability decreased.

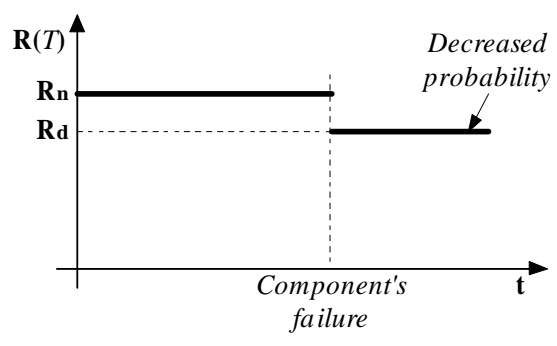

Fig. 3 Decreasing the nominal reliability

Figure 3 shows reliability reduction of the quasi-redundant component during its mission from its nominal reliability level $\left(R_{n}\right)$ to reduced level $\left(R_{d}\right)$. Consequently, the system will follow its primary mission thanks to quasi-redundant controller but its reliability will be decreased. The difference between nominal and reduced reliability level we can sign as decrease factor $d_{R}$. The influence of possible scenarios and decrease factor to final reliability of the studied system is shown in further text.

\subsection{Application to basic NCCS and a mini-drone helicopter}

In this paper, we focus on a basic networked cascade control structure (Fig. 4) as well as mini-drone control structure. The basic NCCS is composed of 6 subsystems: two sensors, two controllers, one actuator and the network, which is the simplest network cascade architecture. This architecture is referred in the following as "basic cascade structure".

The NCC structure is also applied for the control of a four rotors mini-helicopter (Drone, Fig. 5). The proposed control structure for this real model is as follows. The NCC architecture is composed of one primary controller (Master) and one secondary controller (Slave), thirteen sensors, four actuators and two communication networks. 


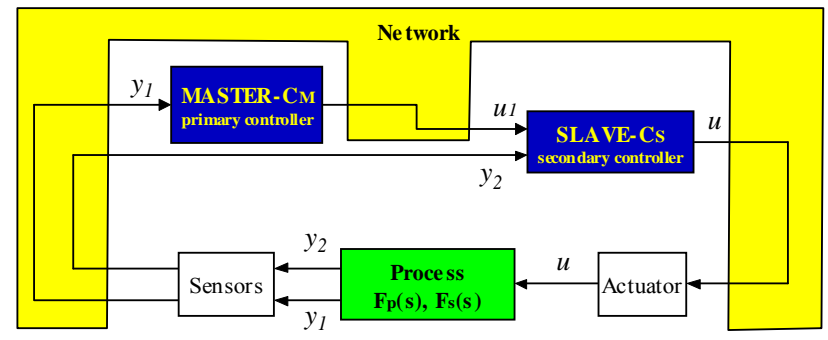

Fig. 4 Structure of the networked cascade control system (NCCS) with one network and two quasi-redundant controllers (primary Master and secondary Slave)

The Master is designed for attitude stabilization (control) through Slave controller for angular velocity control for each propeller. The aim of the control is to stabilize coordinates of the helicopter (Castillo, et al., 2004).

The controllers are used as quasi-redundant components within presented networked cascade control system (further only NCCS). They use the same control algorithm (propeller's angular velocity control) but with different input data (set point, system output, etc.)
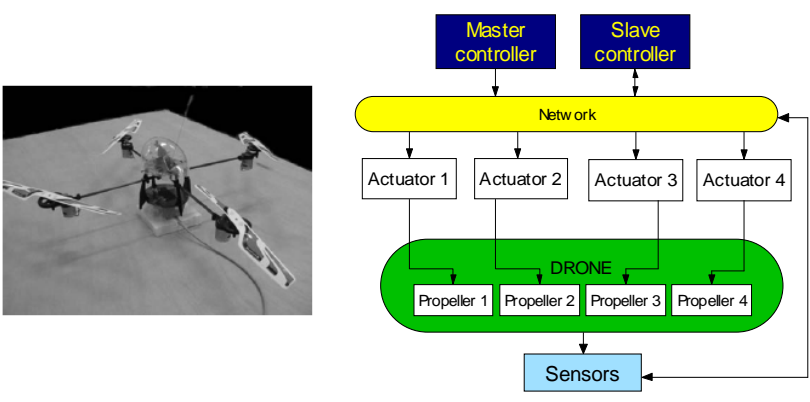

Fig. 5 NCC structure of mini-helicopter with two networks

Hence, in case of failure one of them could retransmit all required data to another one, whereas pre-programmed control algorithm should compute the actuating value. Thus, failed controller is replaced by second one which start to compute actuating value.

Other quasi-redundant parts of this control structure are networks (Fig. 6). The same as in case of controllers, one of the networks can compensate another one after system reconfiguration. Usually, two networks are primary designed due to reduction amount of transmitted data. However, in case of network failure all data could be retransmitted through second one.

Described approach for subsystem's failure compensation by using the shared redundancy requires logical reconfiguration of the NCCS. Thus, in case of failure the hardware configuration is non-touched but communication ways must be changed in order to transmit the data to non-failed component or through non-failed network.

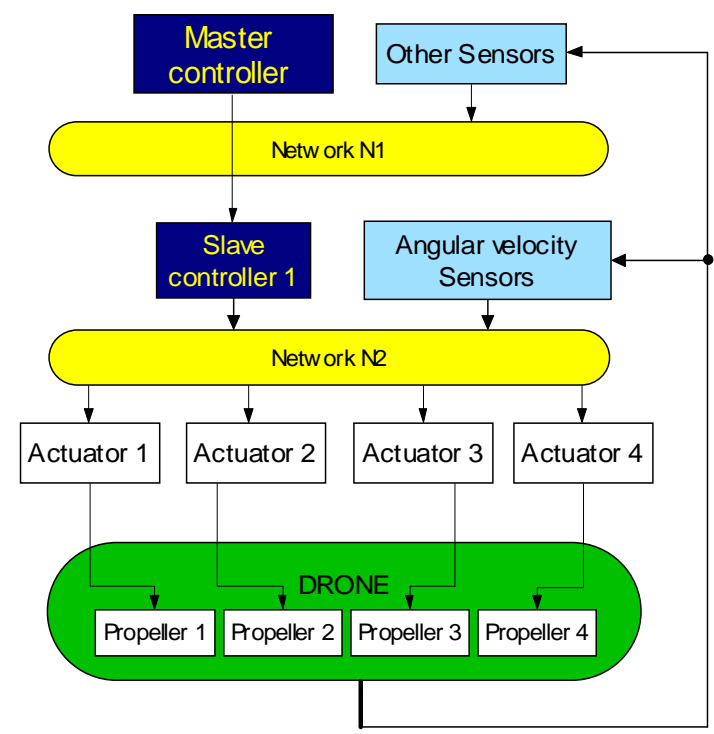

Fig. 6 NCC structure of mini-helicopter with two networks

\section{SIMULATIONS AND RESULTS}

All presented networked control architectures (Fig. 4, 5, 6) were modelled by using Petri nets. This tool was chosen thanks to its ability to model different types of complex systems and dependencies within them. To provide the reliability analysis the Monte Carlo simulation (further only MCS) method was used. The multiple simulations of the modelled architecture (Pimentel et al. 2002) are provided to obtain the reliability curves for all above described NCCS. The MCS is a statistical method where its exactness is significantly influenceable by the number of provided simulations (sample's cardinality). The simulations of the prepared models was provided 50000 times.

Defined nominal reliability $R_{n}(T)$ all of the components included by control system as Sensors, Controllers, Actuators and Networks within one sample period is $R_{n}(T)=0.999$. Thus, probability of failure of the components is $10^{-3}$ for one sample period $T$.

In all simulated systems was observed the influence of the quasi-redundant components to final system reliability. In figure 7 are shown the results of simulations for the basic networked cascade architecture shown in figure 4 . The same as in other figures there are shown the reliability curve of system with quasi-redundant subsystems $\left(\mathrm{d}_{\mathrm{R}}=0\right)$ and without them $\left(d_{R}=0.999\right)$. Between these two values of decrease factor are shown the three curves which represent final reliability of the system with decrease factors as $2.10^{-3}, 10^{-2}$, $59.10^{-3}$. In figures 8 and 9 are shown curves in detail too. We can see that decreasing the component's nominal reliability by decrease factor equal to $59.10^{-3}$ which represents approximately $6 \%$ of nominal reliability $R_{n}$ has significant influence to decreasing the final reliability of the system. The results are a little bit better than in case of system without 
redundant components $\left(d_{R}=R_{n}=0.999\right)$, but we could say that almost the same.

Figures 8 and 9 show simulation results of the drone's cascade control architectures (Fig. 5, 6). The parameters of the system change significantly the complexity of the simulated structure. Compared to basic NCCS (Fig. 4) only 6 components are considered, the drone's structure composes of twenty (twenty-one) components - thirteen sensors (3 gyrometers, 3 magnetometers, 3 accelerometers, 4 rotors' angular velocity sensors), two controllers, four actuators and one (two) networks. This significant change we can see in both figures (Fig. 8,9) where difference between curve for minimal and maximal $d_{R}$ is smaller than in case of basic cascade architecture. On the other side, with regarding on behavior of the system reliability under influence of the decrease factor $d_{R}$ we can see that influence is very similar like in first case shown in figure 7. Likewise, when the decrease factor is set up to $59.10^{-3}$ the final reliability curve of the system was approached to reliability curve of the same system without quasi-redundant components. The comparison of the results all simulated architectures by using other dependability attribute-MTTFF is shown in table 1 .

The Mean Time Before First system's Failure is significantly longer in case of basic NCCS than in drone's cases. As it was mentioned above this is caused by difference in complexity between basic and drone's NCC architecture. In case of comparison between two drones structures (Fig. 5, 6) the results are better for architecture with two networks which is composed of two quasi-redundant subsystems - controllers (Master, Slave) and networks when decrease factor is smaller than $59.10^{-3}$. This decrease factor or greater one gets faster to values given by system without shared subsystems.

\section{Table 1. MTTFF of simulated control structures} with different decrease factor

\begin{tabular}{|c|c|c|c|}
\hline$d_{R}$ & $\begin{array}{c}\text { MTTFF } \\
\text { Basic NCCS } \\
\text { (Fig. 4) }\end{array}$ & $\begin{array}{c}\text { MTTFF } \\
\text { Drone } \\
\text { (Fig. 5) }\end{array}$ & $\begin{array}{c}\text { MTTFF } \\
\text { Drone } \\
\text { (Fig. 6) }\end{array}$ \\
\hline 0 & 236.4 & 54.79 & 57.62 \\
\hline $2.10^{-3}$ & 213.07 & 53.81 & 56.2 \\
\hline $10^{-2}$ & 186.76 & 53.2 & 54.27 \\
\hline $59.10^{-2}$ & 169.04 & 50.49 & 49.18 \\
\hline $\mathrm{R}_{\mathrm{n}}=0.999$ & 167.03 & 49.66 & 47.6 \\
\hline
\end{tabular}

Obtained result describe the influence of the decrease factor which could appear when it is necessary to prevent a component's critical failure by using any component with similar mission but its reliability is reduced by a specified value.

From results we can see that positive influence to reliability increasing using quasi-redundant (shared) subsystems is logically reduced by reducing the ratio between number of non-redundant and redundant components of the studied system.

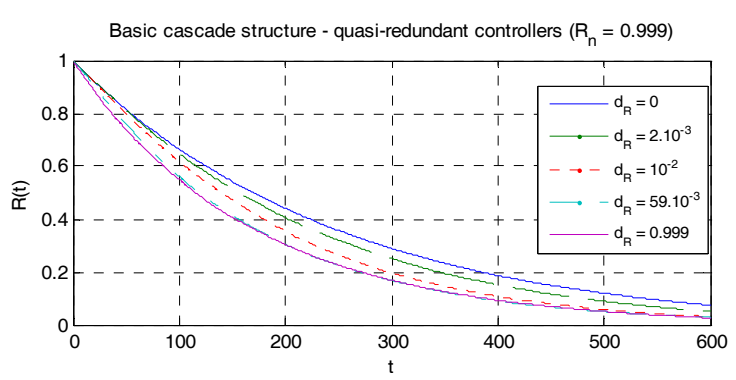

Fig. 7 Simulation results of the basic cascade control architecture with two quasi-redundant controllers
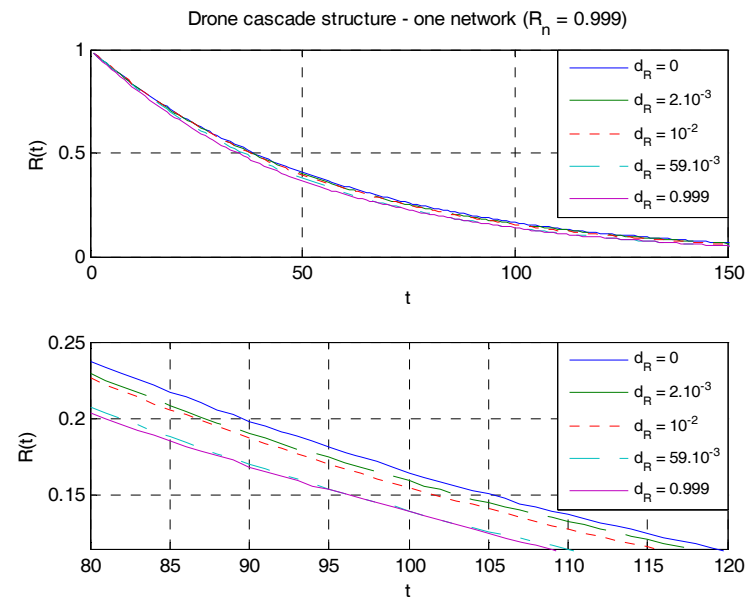

Fig. 8 Simulation results of the mini-helicopter control architecture with quasi-redundant controllers (case with one network only)
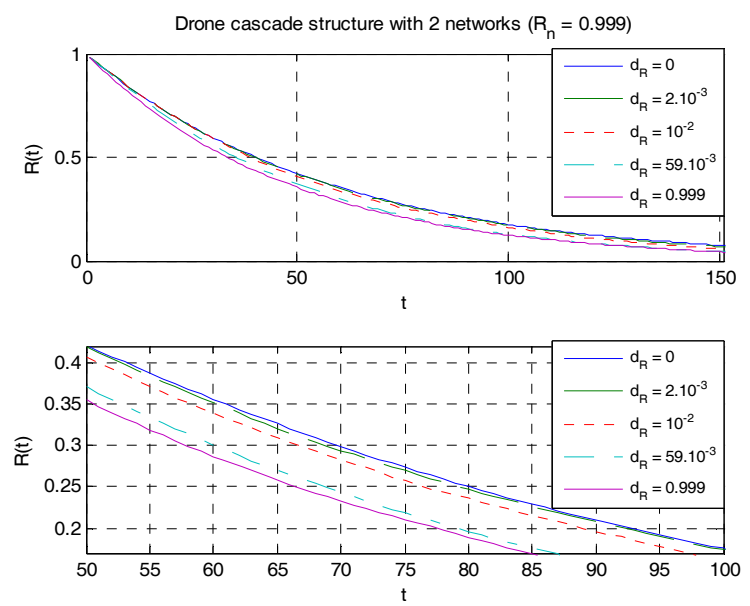

Fig. 9 Simulation results of the mini-helicopter control architecture with two types of quasi-redundant subsystems (controllers, networks) system 
The next observation shows us that the ideal state is when decrease factor is 0 or very small - until $6 \%$ of the nominal component's reliability $\left(\mathrm{d}_{\mathrm{R}} \leq 59.10^{-3}\right)$. When additional reliability reduction is greater than $6 \%$ of nominal reliability value $R_{n}\left(\mathrm{~d}_{\mathrm{R}} \geq 59.10^{-3}\right)$ the system gets to similar reliability level as in case without any redundant subsystem.

\section{CONCLUSIONS}

The paper shows the influence of additional reliability decreasing of the quasi-redundant component to entire reliability of the studied system. Description of this dependency is getting closer to show the behavior of the system reliability when shared redundancy approach is implemented within networked control structure. Presented control architectures could be applied to similar systems. For example, Steer-by-Wire control (Leen et al., 2002, Yih et al., 2005) of two front wheels in a car, etc.

Even if the reliability of the system is significantly decreased (in comparison with the reliability level obtained by conventional active redundancy - see Fig. 7,8,9 curve $d_{R}=0$ ) the implementation of this control architecture which takes into account "source sharing" has a significant contribution especially in case of critical failures. To solve the problems with critical failures it is necessary that control system take the process on safe state. The using of shared redundancy approach could help us to solve this kind of problem too.

In addition the paper has shown the conventional cascade control structure within conditions of networked control systems as naturally suitable to profit from quasi-redundant subsystems as networks, controllers and potentially sensors if physical process allows it. Despite of some constraints for using this type of control is cascade architecture widely used in industrial control applications. Hence, only the reconfiguration algorithm should be implemented to profit from quasi-redundant subsystems.

The main advantages of the quasi-redundant components could be summarized as follows:

- The system composes only of necessary components (parts) for following the primary mission of the system whereas higher system reliability is ensured without using any additional active redundant components.

- Following the first point we could suppose less number of the components used for safe the control mission. Thus, economic aspect could be very significant.

- Prevention of system's critical failure also when subsystems have not sufficient hardware capacities.

\section{REFERENCES}

Brosilow, C., Babu, J. (2002). Techniques of ModelBased Control, Chapter 10, Prentice Hall, ISBN: 9780-13-028078-7
Castillo, P., Dzul, A., Lozano, R. (2004). Real-Time Stabilisation and Tracking of a Four Rotor MiniRotorcraft, IEEE Transaction on control systems technology, Vol 12, No. 4, p. 510 - 516.

Galdun, J., Ghostine, R., Thiriet, J. M., Liguš, J., Sarnovský J. (2007). Definition and modelling of the communication architecture for the control of a helicopter-drone, 8th IFAC Symposium on Cost Oriented Automation

Guenab, F., Theilliol, D., Weber, P., Zhang, Y.,M., Sauter, D. (2006). Fault-tolerant control system design: A reconfiguration strategy based on reliability analysis under dynamic behaviour constraints, p. : 1387-1392, 6th IFAC Symposium on Fault Detection.

Laprie, J. C., Kopetz, H., Avižienis, A. (1992). Dependability: Basic Concepts and Terminology, Chapter 1, Springer-Verlag / Wien, ISBN: 3-211-82296-8

Leen, G., Heffernan, D. (2002). Expanding Automotive Electronic Systems, Computer IEEE, Vol. 35, p.: 88-93

Ligušová J., Thiriet, J.M., Liguš, J., Barger, P. (2004). Effect of Element's Initialization in Synchronous Network Control System to Control Quality, RAMS/IEEE conference Annual Reliability and Maintainability Symposium.

Nicolescu, S.,I. (1997). Stabilité systèmes à retard Aspects qualitatifs sur la stabilité et la stabilisation, Diderot multimedia, ISBN: 2-84352026-6

Pimentel, J.R., Salazar, M. (2002). Dependability of Distributed Control System Fault Tolerant Units, The 28th Annual Conference of the IEEE Industrial Electronics Society, vol. 4, p. 3164- 3169, IECON '02, ISBN: 0-7803-7474-6

Spooner, J. T.,Passino, K.,M. (1997). Fault-Tolerant Control for Automated Highway Systems, IEEE Transactions on vehicular technology, vol. 46, no. 3, p. 770-785

Tanwani, A., Galdun, J., Thiriet, J-M., Lesecq, S., Gentil, S. (2007). Experimental Networked Embedded Mini Drone - Part I. Consideration of Faults, European Control Conference 2007

Wysocki, J., Debouk, R., Nouri, K. (2004). Shared redundancy as a means of producing reliable mission critical systems, 2004 Annual Symposium - RAMS Reliability and Maintainability, p.: 376-381

Yih, P., Gerdes, J.C. (2005). Modification of Vehicle Handling Characteristics via Steer-by-Wire, IEEE Transactions on Control Systems Technology, Vol. 13, p. $965-976$ 